\title{
Utilización de documentos históricos en la reconstrucción de la vegetación de la Llanura de la Travesía (Argentina) a principios del siglo XIX
}

\author{
The use of historical documents to reconstruct the vegetation of the Llanura \\ de la Travesía (Argentina) during early nineteenth century
}

\author{
MARÍA DEL ROSARIO PRIETO ${ }^{1}$, PABLO EUGENIO VILLAGRA $^{1}$, \\ NERINA BELÉN LANA ${ }^{2} \&$ ELENA MARÍA ABRAHAM ${ }^{3}$
}

\author{
${ }^{1}$ Departamento de Dendrocronología e Historia Ambiental, Instituto Argentino de Nivología, Glaciología y Ciencias \\ Ambientales, Casilla de Correo 330, 5500 Mendoza, Argentina; e-mail: mrprieto@lab.cricyt.edu.ar \\ ${ }^{2}$ Universidad de Congreso, Colón 90, 5500 Mendoza, Argentina \\ ${ }^{3}$ Instituto Argentino de Investigaciones de las Zonas Áridas, Casilla de correo 507, \\ 5500 Mendoza, Argentina
}

\begin{abstract}
RESUMEN
La Llanura de la Travesía se extiende en las provincias de Mendoza y San Luis (Argentina) entre el piedemonte de los Andes y las Sierras Pampeanas. El objetivo de este trabajo fue reconstruir las condiciones ambientales de la Llanura de la Travesía a principios del siglo XIX combinando la información de diversas fuentes documentales. Se utilizó como base un testimonio del Comandante Don Faustino Ansay, levantado en 1802 ante la necesidad de verificar el estado del camino de la Travesía o camino Real que unía Corocorto (actual Villa de La Paz) con San Luis. Esta información se complementó con el testimonio de otros viajeros que recorrieron el camino. Este análisis reveló un gradiente similar al actual, en términos de un aumento de la complejidad de la vegetación en el sentido oeste-este. Sin embargo, la ausencia de Aspidosperma quebrachoblanco sugiere cambios en la distribución de algunas especies que deberán ponerse a prueba con nuevas observaciones. Se comprueba la validez de la utilización de información documental para reconstruir la vegetación del pasado y detectar cambio ambientales.
\end{abstract}

Palabras clave: historia ambiental, "Monte", espinal, reconstrucción de la vegetación.

\begin{abstract}
The Llanura de la Travesía extends in the Mendoza and San Luis provinces between the Andean piedmont and the Sierras Pampeanas. The goal of this study was to reconstruct the vegetation and soil characteristics of the Llanura de la Travesía during early 1800 s by means of a combinationg of information from several documentary sources. The reconstruction was based mainly on the reports of Don Faustino Ansay, recorded in 1802 to verify the state of the Camino de la Travesía or Real (Royal Road) from Corocorto (present Villa de la Paz) to San Luis. This information was complemented with field notes from other travelers along the road. Our results revealed that plant cover and density increased from west to east, as it is observed today. However, the absence of Aspidosperma quebracho-blanco in the Ansay's report, suggests that changes in the distribution of some tree species have occurred. This study validates the use of documental information to reconstruct past vegetation and soil characteristics. This information can be used as a valuable tool to detect environmental changes.
\end{abstract}

Key words: environmental history, Monte desert, reconstruction of vegetation.

\section{INTRODUCCIÓN}

Los acelerados procesos de cambio ambiental que están ocurriendo en la actualidad son consecuencia de procesos antrópicos originados en la mayor demanda de recursos naturales y en la producción industrial y agropecuaria. Diversos estudios han intentando cuantificar la magnitud de estos cambios (Skole \& Tucker 1993, Mannion 1997, Wolters et al. 2000). Sin embargo, no siempre se conoce el estado inicial con el cual comparar las condiciones actuales. En este contexto, la reconstrucción de la vegetación del pasado a través de fuentes históricas documen- 
tales puede ser complementaria, desde el punto de vista de su escala temporal y de su resolución espacial, a otras como las fotografías aéreas, la repetición de relevamientos anteriores (Buffington \& Herbel 1965), o los registros palinológicos, "pack rats" y dendrocronológicos (Bradley 1985).

Uno de los aportes fundamentales de las fuentes históricas es su escala temporal, frecuentemente de mayor amplitud que la de los registros instrumentales. Por lo general, en sitios donde no se cuenta con un registro científico de larga data, las descripciones de vegetación existentes no representan la condición inicial. Solo en algunos lugares se dispone de descripciones detalladas que se extienden más allá de cien años, lo que permitiría un análisis claro de los cambios ambientales. En este contexto, la reconstrucción histórica en combinación con la interpretación fitosociológica de remanentes y ambientes degradados permitirían estimar la dinámica de los cambios en la vegetación con cierto grado de certeza.

El ambiente de la gran planicie que se extiende al oriente de la zona cultivada del norte de Mendoza, conocida como Llanura de la Travesía, presenta en la actualidad evidentes signos de degradación ambiental. La tala indiscriminada de los bosques de Prosopis flexuosa (algarrobales) que poblaban el área es mencionada generalmente como uno de los factores desencadenantes del proceso (Abraham \& Prieto 1999). Este proceso comienza a agudizarse a fines del siglo XIX como consecuencia de la sobreexplotación de los bosques para responder a las demandas de la industria vitivinícola y de una población urbana creciente (Abraham \& Prieto 1999).

Numerosas descripciones de viajeros y naturalistas, provenientes de documentos de archivo, sugieren que los bosques de algarrobo cubrían en forma discontinua la planicie. Si bien esta información se limita a las zonas más cercanas al antiguo Camino Real o de la Travesía (actual Ruta 7), se puede asumir la existencia de las mismas formaciones en el resto del territorio. Según Prieto \& Wuilloud (1986), el algarrobo de porte arbóreo no era común en el área donde se fundó la ciudad de Mendoza en 1561, en la línea de confluencia entre el piedemonte y la planicie. Una prueba de ello es que a fines del siglo XVII, cuando los pobladores necesitaban madera para la construcción de viviendas se recurría a los algarrobales de San Luis y de las inmediaciones de las lagunas de Guanacache. Esto último ha permitido ubicar el comienzo del bosque hacia el norte, a unos 20-25 km del área fundacional. Hacia el este, de acuerdo con el Padre Miguel Herre (Muhn 1946) a mediados del siglo XVIII, los “... bosques, selvas y matas..." comenzaban en la ciudad de San Luis y disminuían casi hasta desaparecer unos $25 \mathrm{~km}$ antes de llegar a la ciudad de Mendoza. No obstante estos antecedentes, las referencias no son lo suficientemente concretas como para obtener un conocimiento detallado de la vegetación del área. Pocos viajeros han mencionado las especies que se presentaban a su vista y, en general, es dudosa la localización exacta de las observaciones.

El objetivo de este trabajo es reconstruir las condiciones ambientales de la Llanura de la Travesía a principios del siglo XIX combinando la información de diversas fuentes documentales. Se enfatiza especialmente la presencia de bosques de algarrobos, su extensión y su distribución en el área.

\section{MATERIALES Y MÉTODOS}

\section{Área de estudio}

La Llanura de la Travesía forma parte de una profunda cuenca sedimentaria que se extiende en territorios de las provincias de San Luis y Mendoza (Fig. 1), entre dos bloques montañosos: la cordillera y la precordillera andina hacia el oeste y las Sierras Pampeanas Occidentales en el este (Abraham 2000). El límite sur está dado por el territorio volcánico de la Payunia. Hacia el sureste se conecta con la región pampeana a través de la Pampa Ondulada.

Climáticamente, la llanura presenta un gradiente de precipitaciones desde $150 \mathrm{~mm}$ anuales al oeste a $400 \mathrm{~mm}$ al este, con temperaturas medias que oscilan alrededor de $\operatorname{los} 17^{\circ} \mathrm{C}(\mathrm{Ca}-$ bido et al. 1993). Como respuesta a este gradiente, la vegetación se caracteriza por un enriquecimiento estructural y de especies con el aumento de las precipitaciones; el número de estratos y la cobertura vegetal aumentan y, en consecuencia, la fisonomía característica pasa de una estepa arbustiva en el oeste a bosques cerrados y continuos en la zona más húmeda (Cabido et al. 1993).

En la llanura mendocina dominan distintas comunidades arbustivas xerofíticas de hojas perennes (Roig 1981). La única especie que llega a formar bosques aislados es el algarrobo dulce (Prosopis flexuosa) acompañado por otros árboles como Geoffroea decorticans (chañar) y Bulnesia retama (retamo). Las especies arbustivas más frecuentes son Larrea divaricata y $L$. cuneifolia (jarillas), Capparis atamisquea (atamisque), Condalia microphylla (piquillín), 


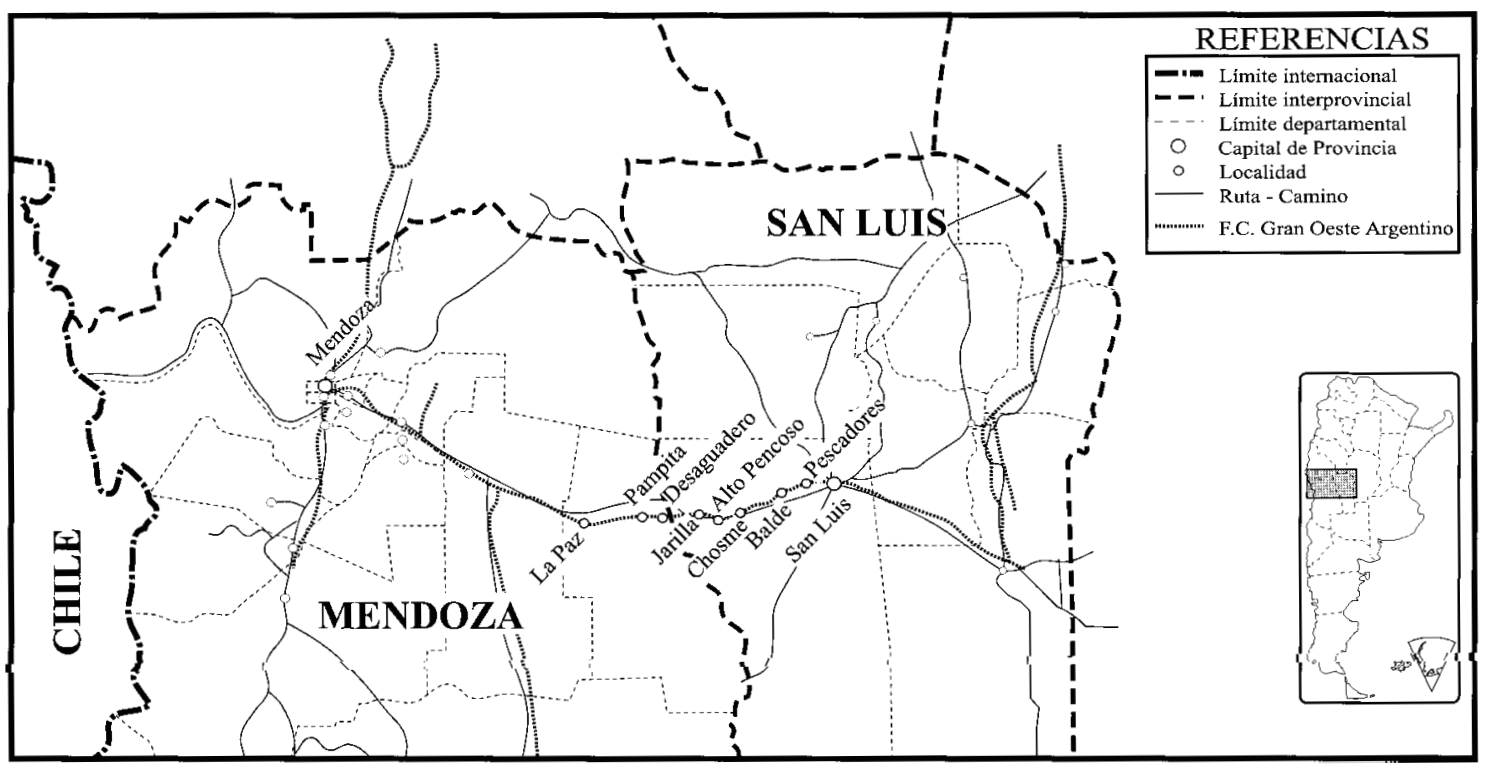

Fig. 1: Mapa actual con las principales localidades de la zona de estudio.

Present map with the main localities of the study zone.

Atriplex lampa (zampa) y Bougainvillea spino$s a$. El porcentaje de suelo desprovisto de vegetación oscila entre 20 y 40 \% (Roig 1981, Guevara et al. 1997). Al este del río Desaguadero, el número de especies chaqueñas aumenta, así también como el número de especies deciduas. En el estrato arbóreo, Aspidosperma quebracho-blanco (quebracho blanco) se hace dominante junto con Prosopis flexuosa, mientras que en el estrato arbustivo aparecen Acacia aroma (tusca), Acacia caven (aromo), Mimozyganthus carinatus, Aloysia gratísima y Lippia turbinata (Roig 1981, Cabido et al. 1993).

El área considerada en este trabajo se extiende desde la localidad de La Paz, en la Provincia de Mendoza hasta la ciudad de San Luis (Fig. 1). En ella, se han distinguido varias subunidades: la planicie terminal del río Tunuyán, caracterizada por bosques abiertos con estratos arbustivos y herbáceos desarrollados; la planicie del río Desaguadero, caracterizada por una estepa arbustiva halófita con sectores de bosques abiertos; y la planicie oriental al norte del río Tunuyán, caracterizada por bosques abiertos que se alternan con estepas arbustivas (Braun et al. 1984). Cruzando el río Desaguadero hacia el oriente, predominan los bosques semicerrados y cerrados con estrato arbustivo desarrollado.

\section{Fuentes documentales}

Para conocer la distribución del bosque en el área a comienzos del siglo XIX se ha utilizado básicamente el testimonio del Comandante Don Faustino Ansay, quien, en 1802, fue comisionado para examinar el estado del Camino Real o de la Travesía en el tramo que unía la Villa de Corocorto y la ciudad de San Luis. Se trataba de comparar las cualidades de los tres caminos trazados entre estos dos puntos para decidir cuál era el más conveniente para el tránsito de carretas. En el "Plano de los caminos contratados entre el gremio de carreteros de Mendoza y don Francisco Serra Canals" 1 realizado en 1803, están localizadas las tres vías utilizadas por los carreteros en ese período (Fig. 2). Para informar acerca de las condiciones de estos caminos, Ansay elaboró tres diarios, depositados actualmente en el Archivo Histórico de Mendoza $(\mathrm{AHM})^{2}$. La vegetación de la Travesía del Medio o Camino Real, objeto de este estudio, se describe en el segundo diario. El documento describe detalladamente la vegetación y el suelo que iba atravesando, por tramos de entre 100 y 300 varas (entre 80 y $240 \mathrm{~m}$ ).

El denominado "Camino Real o de la Travesía”, unía el Atlántico con el Pacífico. Saliendo

\footnotetext{
${ }^{1}$ Mapas y Planos, Buenos Aires 210, Archivo General de Indias (AGI).

2 "Diario de lo que se ha inspeccionado en el Camino del Bebedero Año de 1802", Colonial, C 34/ D 16, 1802, AHM; "Diario de lo que se ha inspeccionado en el Camino de la Travesía del Medio, Año de 1802". Colonial, C 34/ D 15, año 1802, AHM. "Diario de lo que se ha inspendido en el Camino de las Lagunas", Colonial, C 34/ D 14, 1802, AHM.
} 


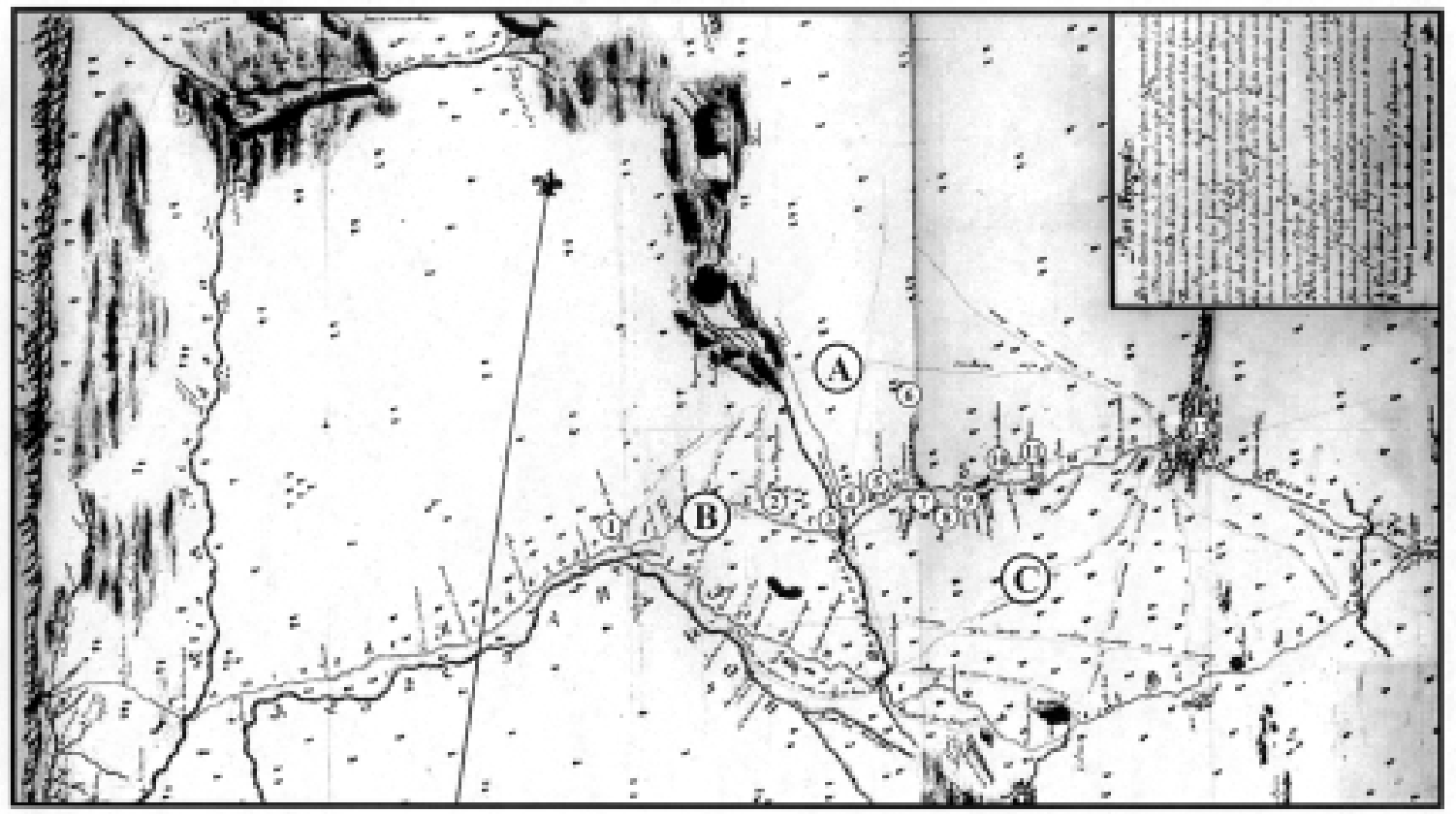

Fig. 2: En base al "Plano de los caminos contratados entre el gremio de carreteros de Mendoza y don Francisco Serra Canals" realizado en el año 1803, se resaltan los tres caminos principales utilizados por los carreteros en ese período y las localidades mencionados por Don Faustino Ansay en su documento: (A) Camino de los Arrieros, (B) Camino de la Travesía, Real o del Medio (era el más transitado), y (C) Camino del Bebedero. (1) Villa de Corocorto (actual Villa de La Paz), (2) Barriales de los Algarrobos, (3) Puente Desaguadero, (4) Patito Parado, (5) Rodeito de Poveda, (6) Cerrilladas del Portezuelo, (7) Pozo del Milagro, (8) Los Pejes, (9) Alto del Yeso, (10) Alto de las Palomas, (11) Corral de los Chomes, (12) Rodeo de las Catitas, (13) San Luis.

Based on the "Map of the roads engaged between the cart drivers union and Don Francisco Serra Canals" drawn in 1803 , we stand out the three main roads used by the cart drivers during that period and the main localities mentioned by Ansay in his report: (A) Camino de los Arrieros, (B) Camino de la Travesía, Real o del Medio, and (C) Camino del Bebedero. (1) Villa de Corocorto (actual La Paz), (2) Barriales de los Algarrobos (3) Puente Desaguadero, (4) Patito Parado, (5) Rodeito de Poveda, (6) Cerrilladas del Portezuelo, (7) Pozo del Milagro, (8) Los Pejes, (9) Alto del Yeso, (10) Alto de las Palomas, (11) Corral de los Chomes, (12) Rodeo de las Catitas, (13) San Luis.

de Buenos Aires, atravesaba Córdoba, San Luis y Mendoza, cruzaba la Cordillera de los Andes e ingresaba a Chile y desde allí se conectaba con Perú por mar o por tierra. Se estima que unas 1.200 carretas transitaban anualmente por este camino hacia 1800 , el cual era una vía obligada para la colocación de los productos de Mendoza en las ciudades situadas al oriente (Prieto \& Abraham 2000).

Por otra parte, las características del camino estimularon también a muchos viajeros que hacían la travesía entre Buenos Aires y Santiago a describir el recorrido realizado. De ellos ha quedado una extensa variedad de relatos que han sido utilizados complementariamente en este trabajo (e.g., Haenke 1794, Macrae 1856, Caldcleugh 1943, Schmidtmeyer 1947, GreeneArnold 1951).

\section{Procesamiento de la información}

Para reconstruir la cobertura vegetal y el suelo de la planicie de principios del siglo XIX se utilizó el método fisonómico que se fundamenta en la identificación de grandes unidades de vegetación tal como se presentan o impresionan al observador, teniendo en cuenta la estructura de la vegetación y la o las especies dominantes (Kent \& Coker 1992). Se asumió que el observador del pasado realizaba espontáneamente este mismo ejercicio. De este modo, se asumió que los observadores no expertos en botánica registraban los elementos del paisaje prestando mayor atención a la configuración global de la vegetación que a las especies en particular. Así, se ha verificado que predominan los conceptos de algarrobales, 
jarillales o chañarales en lugar de términos específicos para denominar las especies de algarrobo, jarilla o chañar.

\section{Desarrollo de categorías de análisis a partir} del texto y equivalencias de significado

Por otra parte, se verificó el uso de una multiplicidad de términos en los diarios para designar a cada formación identificada. Para reducir la cantidad de expresiones referidas a cada tipo de vegetación, y obtener datos homogéneos, se aplicó a los textos la técnica lingüística conocida como "análisis de contenido" (Baron 1982). El objeto fue transformar en conceptos científicos las nociones vulgares transmitidas por el observador del pasado. Esta técnica permite establecer y calibrar el significado de las expresiones presentes en los documentos referidos al ambiente, verificar la permanencia de términos y expresiones sobre elementos del ambiente a través del tiempo, establecer categorías en relación con la vegetación o la fauna y calificar los fenómenos de acuerdo a su magnitud, estableciendo un rango entre fenómenos de la misma naturaleza (Baron 1982).

En primer lugar se procedió a la identificación de todos los términos utilizados para describir la vegetación registrada por Ansay a lo largo del camino. Se extrajo el significado de cada término utilizado a partir de diccionarios de la época como el "Diccionario de la Lengua Castellana compuesto por la Real Academia Española reducido a un tomo para su más fácil uso" (Real Academia Española 1791).

\section{Caracterización del término "monte"}

Se verificó que el término "monte" era la expresión más reiterada por Ansay para describir la vegetación. Esto condujo a realizar un estudio particularizado de este vocablo. En primer lugar se verificó si existía una correspondencia entre el significado del término en el presente y en la época en que se realizó el transecto. Según Font-Quer \& Sierra Rafols (1979), el término "monte" equivale, con poca precisión, a formación leñosa, llamándose "monte bajo" si se trata de matorral o fruticetum y "monte alto" si de una formación arbórea. En los Diarios se remarca la espesura del monte que se debía atravesar entre el río Desaguadero y Corocorto: “...hay en el campo bastante pasto [...] pero hay bastantes retazos de monte espeso y como se halla el camino abierto por entre el dicho monte, está el piso con mucha tronquería, lo mismo de reigones y varios pedazos de tunas junto al camino, sien- do el monte como llevo dicho, espeso..."3. De acuerdo con este párrafo, el concepto de monte se acercaría más a un estrato arbóreo bastante desarrollado que a un arbustal.

Para determinar si en el siglo XVIII la palabra monte tenía el mismo significado en América que en España, se acudió al diccionario mencionado anteriormente (Real Academia Española 1791), monte es "la tierra cubierta de árboles, que llaman "monte alto", o de malezas, que llaman "monte baxo". "Monte cerrado" (o moeda) es "el encinar viejo, espeso y cerrado de maleza..." (p. 574). Se observa que el sentido de la expresión es el mismo que el otorgado por FontQuer \& Sierra-Rafols (1979). Por lo tanto es posible equiparar la definición anterior con la de monte espeso de los Diarios de Ansay.

Se deduce entonces que el término monte surgió en España y alude específicamente a especies originarias de la Península Ibérica, como la encina. El término fue trasladado a América, donde se lo aplicó a las formaciones arbóreas americanas con el mismo sentido, perdurando hasta la actualidad. Una afirmación de Lemos (1888), a fines del siglo XIX, ratifica esta tesis cuando refiriéndose a la vegetación de Mendoza dice que "Las especies vejetales...se encuentran esparcidas en todas las comarcas ya formando agrupaciones o bosques llamados vulgarmente montes" (Lemos 1888, p. 104), lo que implica en este caso, una asociación de árboles (algarrobos) y arbustos de distinto tamaño y densidad.

\section{Determinación de equivalencias de significado}

Ansay, además de la palabra "monte", utilizó varios términos similares entre sí para referirse al mismo fenómeno. Para obtener una reducción significativa del número de descriptores se procedió a reunirlos en grandes categorías de acuerdo a su equivalencia de significado. Se tomó como base los patrones que rigen actualmente para la descripción de la vegetación y las descripciones actuales de la vegetación de la zona (Roig 1971, 1981). Las expresiones de Ansay fueron reducidas así a siete categorías que corresponden a distintos porcentajes de cobertura y distribución de las especies: (1) campo desnudo: menos del $20 \%$ de cobertura, con arbustos aislados y muy baja cobertura de pastos; (2) pastizal: coberturas entre 40 y el $70 \%$, alta dominancia de pastos, presencia de arbustos aislados; (3) estepa arbustiva de Larrea

\footnotetext{
3 "Diario de lo que se ha inspeccionado en el Camino de las Lagunas”, Colonial, C 34/ D 14, 1802, AHM, foja 1.
} 
spp.: coberturas entre el 20 y $60 \%$, alta dominancia de arbustos del género Larrea. Estrato herbáceo que varía entre 10 y $30 \%$ de cobertura. En suelos arenosos es dominada por Larrea divaricata y en suelos franco arcillosos por $L a$ rrea cuneifolia. (4) Monte abierto con árboles aislados: estepa de arbustos de diversas especies con árboles aislados. Cobertura de arbustos entre el 20 y $40 \%$; (5) monte semidenso: bosque abierto con un estrato arbustivo denso arbustos de diversas especies. Cobertura de árboles cercana al $20 \%$ y de arbustos entre el 40 y $70 \%$; (6) monte denso dominado por algarrobos: bosque de Prosopis flexuosa con cobertura total superior al $70 \%$ y de árboles superior al $30 \%$. Alta densidad de arbustos de diversas especie. (7) Bosque cerrado de algarrobos: cobertura de algarrobos superior al $50 \%$.

A continuación se buscaron, entre las expresiones que aparecen en el diario de Ansay, aquellas que presentaran similitud de significa- do con cada una de las categorías establecidas, ubicándolas dentro de la categoría correspondiente (Tabla 1).

\section{Tipos de suelo}

El mismo tipo de análisis se aplicó a la información obtenida sobre suelos. Sin embargo, la descripción edáfica realizada por Ansay (1802) es menos minuciosa que la que hace de la vegetación. En la Tabla 2 se detallan las categorías de suelos establecidas y las equivalencias de significados.

\section{Reconstrucción ambiental}

La información fue analizada considerando el camino como un transecto en el que se estimaron las distancias de intercepción de cada tipo de vegetación y de suelo, y se calculó la proporción ocupada por cada uno (Matteucci \& Colma 1982).

TABLA 1

Equivalencias de significado para cada categoría de vegetación detectada Equivalence of meanings for each detected vegetation category

\begin{tabular}{|c|c|c|c|c|c|c|c|}
\hline $\begin{array}{l}\text { Tipo de } \\
\text { vegetación }\end{array}$ & $\begin{array}{c}\text { Campo } \\
\text { desnudo }\end{array}$ & Pastizal & $\begin{array}{l}\text { Estepa arbustiva } \\
\text { de Larrea sp. }\end{array}$ & $\begin{array}{l}\text { Monte abierto } \\
\text { con árboles } \\
\text { aislados }\end{array}$ & $\begin{array}{c}\text { Monte } \\
\text { semidenso }\end{array}$ & $\begin{array}{l}\text { Monte denso } \\
\text { dominado por } \\
\text { algarrobos }\end{array}$ & $\begin{array}{l}\text { Bosque } \\
\text { cerrado de } \\
\text { algarrobos }\end{array}$ \\
\hline Equivalencias & Camino o campo: & "Pastizal" & Jarillal: & Monte: & Monte: & Monte: & "Algarrobal" \\
\hline \multirow[t]{5}{*}{ de significado } & "raso", & "Pastoso" & "espeso" o & "ralo" & "entre espeso", & "espeso", & \\
\hline & "escampado", & "Monte ralo & "ralo" & "claro" & "entre ralo y espeso", & "abundante", & \\
\hline & "ralo", & y campo pastoso" & & "entreclaro" & "algo espeso" & "bastante espeso", & \\
\hline & "limpio", & "Jarillal ralo & & "muy ralo" & "no muy ralo", & "monte bosque", & \\
\hline & "claro" & y campo pastoso" & & & "Monte de chañares" & "monte regular". & \\
\hline
\end{tabular}

TABLA 2

Equivalencias de significado para cada categoría de suelo detectada

Equivalence of meanings for each detected soil category

\begin{tabular}{|c|c|c|c|c|}
\hline Médano & Arenoso & Franco & Cárcavas & Arcilloso \\
\hline \multirow[t]{6}{*}{ "Médano" } & "Arenoso" & "Franco" & "Cárcavas" & "Playones" \\
\hline & "Blando" & "Piso" & "Escorrentía" & "Arcillosos" \\
\hline & & "Bueno" & "Zanjeado" & "Barrial" \\
\hline & & "Firme" & "Quebrado" & \\
\hline & & "Duro" & "Huellas profundas" & \\
\hline & & & "Estrecho" & \\
\hline
\end{tabular}




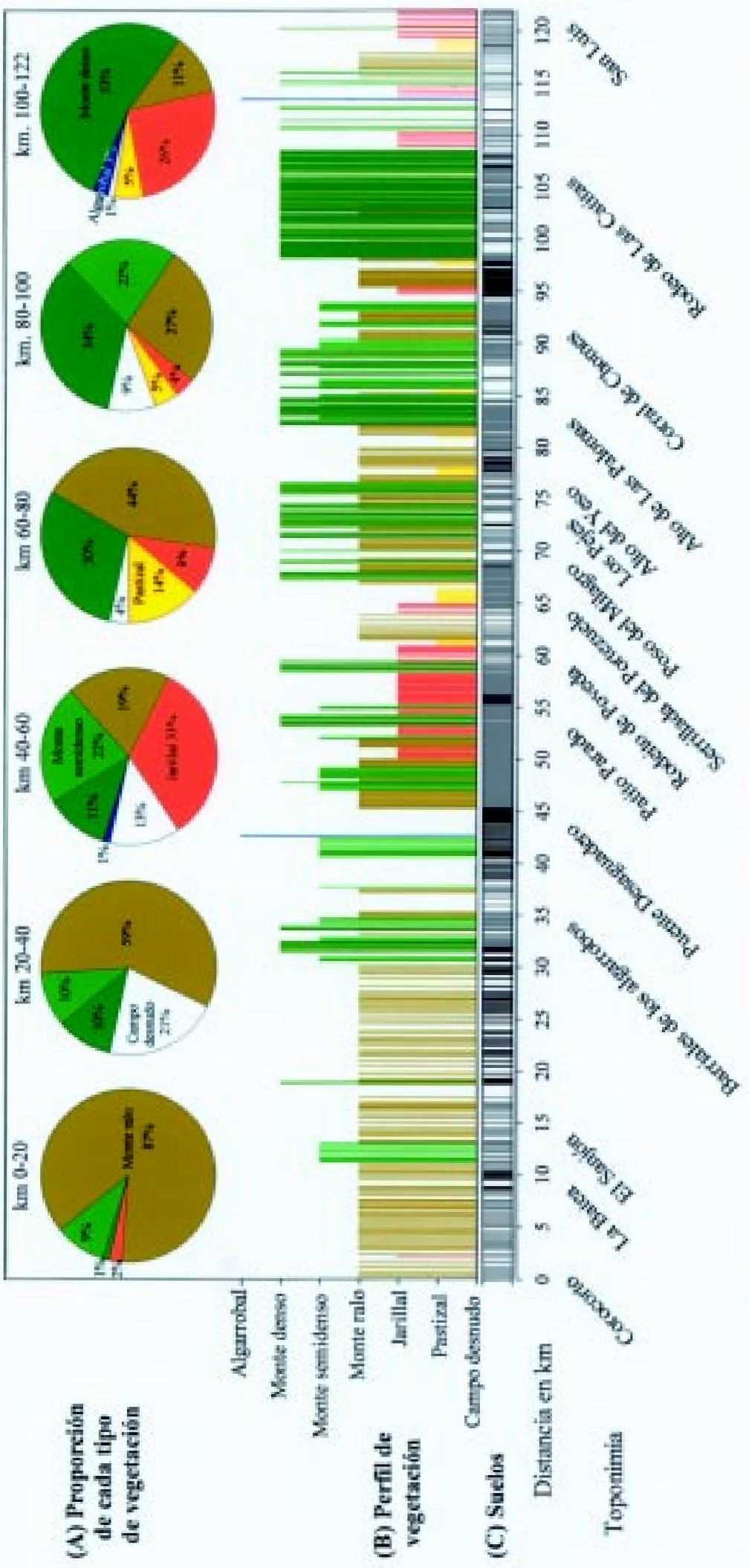

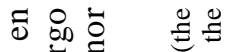

흥 छ

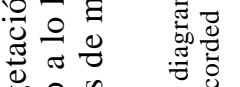

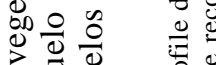

过若

ฮี

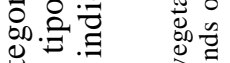

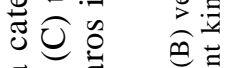

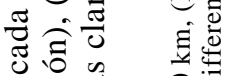

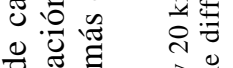

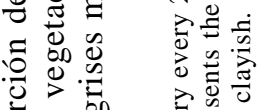

势战

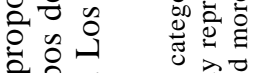

영

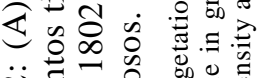

$\ddot{\delta} \cdot \stackrel{0}{0} 0$

$\infty$ :

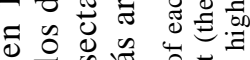

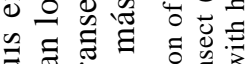

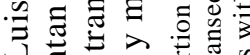

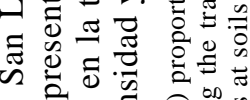

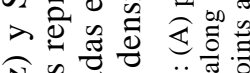

สิ

2. $=$

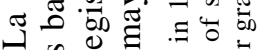

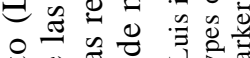

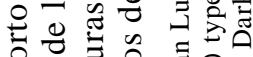

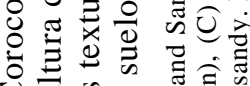

ठ

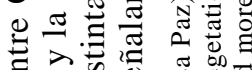

वै

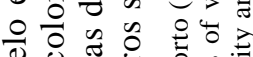

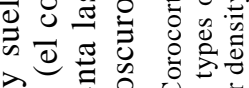

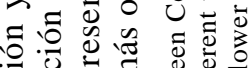

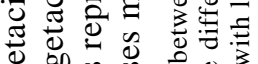

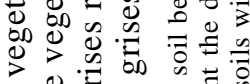

क षे की

음

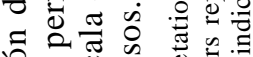

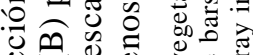
过

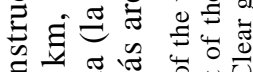

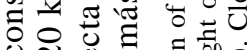
ठㅇ $\simeq$ 응

$\ddot{n} \ddot{\circ}$ 要

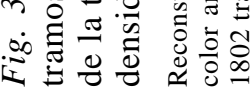


Para realizar un tratamiento gráfico de los datos, a cada categoría de vegetación se le asignó un valor en un rango de 0 a 6 , de acuerdo a las diferencias de biomasa relativa entre las unidades de vegetación. Para los distintos tipos de suelo se utilizó una escala de grises donde los más claros representan suelos más arenosos y los más oscuros aquellos más arcillosos.

\section{RESULTADOS Y DISCUSIÓN}

La vegetación en el transecto Corocorto-San Luis de 1802 presenta un patrón fisonómico similar al actual, con una complejidad creciente hacia el este, donde aumenta la cobertura de especies arbóreas y disminuye la dominancia de las especies arbustivas (Fig. 3). En los $40 \mathrm{~km}$ más occidentales, la unidad dominante era el monte ralo (87 \%). Entre los 40 y $80 \mathrm{~km}$ había mayor heterogeneidad, donde la dominancia del jarillal se alternaba con los montes ralo, semidenso y denso. Por último, en los $20 \mathrm{~km}$ más cercanos a San Luis la formación más abundante era el monte denso (60\%), aunque en los alrededores de esta localidad el jarillal también era muy abundante (Fig. 3 y 4). El jarillal era muy abundante en la zona central del transecto, lo que coincidía con zonas de suelos de textura fina y arcillosa.

La porción mendocina del transecto presentaba una mayor proporción de suelos arenosos mientras que la porción localizada al este del Desaguadero presentaba una mayor proporción de suelos intermedios (seguramente francos como en la actualidad). Los alrededores del río Desaguadero presentaban una alta proporción de superficie ocupada por barriales, constituidos por suelos de textura muy fina, arcillosos (Fig. 3).

Si bien la descripción de Ansay no es muy precisa en cuanto a las especies vegetales que integran cada unidad de vegetación, sí menciona las especies dominantes. Entre estas, el algarrobo (Prosopis flexuosa) era la especie dominante del estrato arbóreo. Sin embargo, en algunas situaciones la especie dominante era el chañar (Geoffroea decorticans), especialmente en zonas de acumulación de agua y suelos arcillosos. Entre las especies del estrato arbustivo se señala a la jarilla como la más abundante. Ansay probablemente incluía como jarilla a las dos especies presentes actualmente, Larrea divaricata y L. cuneifolia. Esta última debió ser la dominante en el jarillal asociado a suelos arcillosos o franco-arcillosos tal cual se puede observar en la actualidad (Roig 1971, Roig \& Rossi 2001). Otras especies arbustivas impor- tantes eran la zampa (Atriplex lampa) y el piquillín (Condalia microphylla). A lo largo de este recorrido también se mencionan a la aparición de tunas, término que puede haber reunido a varias especies de cactáceas.

Las afirmaciones de Ansay son corroboradas por viajeros y naturalistas contemporáneos, algunos de los cuales entregan datos más precisos sobre las especies observadas. Todos los observadores coincidían en que las cercanías de Corocorto presentaban un algarrobal más o menos denso, con escaso estrato arbustivo, grandes espacios de suelo desnudo y, en algunos sitios cercanos a los asentamientos, bosquecillos de "algarrobos enanos" (Prieto \& Abraham 2000). Tadeo Haenke (1794), por ejemplo, afirma que el camino a Corocorto es bueno “... los árboles y arbustos, matas más abundantes y por intervalos. Hay dos paradas buenas [...] la primera es el Zanjón, dista cuatro leguas de la posada no es abundante de agua pero hay un bosque muy frondoso [...] la segunda El Algarrobo son nueve leguas de la referida posada, es sitio más ameno que el Zanjón, abundante de agua..." (p. 37). Sobre el camino de Desaguadero a La Punta, este observador señala que el monte era más frondoso que en el tramo Corocorto-Desaguadero (p. 38), advirtiendo que las especies son otras “....se hallan una Caesalpinia poinciana [actualmente Caesalpinia gilliesi], dos especies de Xanthium [en la zona existen actualmente $X$. ambrosioides, $X$. cavanillesii y $X$. spinosum var. Spinosum], Chenopodium argemone [no corresponde a ningún nombre científico válido], tres especies de Mimosas y son las que más abundan” (p. 39 y 39v).

Por su parte Schmidtmeyer en el año 1820 corrobora lo anterior (Schmidtmeyer 1947). Luego de atravesar San Luis, camino al Desaguadero, este observador afirma que “...continuamos pasando a menudo por algunos montecitos, principalmente de espinos (Acacia spp.) y algarrobos mezclados con el chañar y el poleo (Lippia turbinata) [...] el color del algarrobo es de un agudo verde claro pero su follaje es tan pequeño y escaso, que es dominado por el color pardo oscuro de sus ramas. Es del tamaño de un pequeño manzano..." (p. 165). Unos kilómetros al oeste del río Desaguadero afirma que “... gradualmente las plantas de pasto en forma de matas habían desaparecido y la vegetación consistía principalmente de una cubierta rala de algarrobos, espinos, mirtos [de acuerdo a la descripción podría ser Capparis atamisquea] y algunos otros pequeños árboles y matorrales [...] A veces el suelo era tan nivelado y tan desnudo de plantas chicas, que matorrales un poco más verdes que los otros, tenían la apa- 
riencia de montecillos reciente y cuidadosamente cavados" (p. 170).

Una de las descripciones más ajustada y rica en información es la del Teniente Archibald Macrae (1856), integrante de la United States Naval Astronomical Expedition to the Southern Hemisphere, quien en 1852 realizó un viaje de estudio desde Chile, atravesando la Llanura de la Travesía. En el tramo del camino que interesa, Macrae remarca la existencia de bosques de chañar, algarrobo, retamo (Bulnesia retama) y de montes bajos de jarilla. Respecto al retamo, actualmente muy abundante, su referencia es una de las pocas encontradas sobre esta especie en esta zona de la llanura: "Es llamado "travesía" o lugar donde no hay agua usualmente; [...] Tierra boscosa con chañares, retamos, y algarrobas..." (p. 24). Un poco más adelante este observador insiste sobre la composición de la vegetación: "Desde Mendoza hasta pocas leguas pasando San Luis la región presenta un monte ralo con algarrobas, chañares, retamos y -donde hay monte bajo- jarillas" (p. 52).

En el tramo Desaguadero-San Luis el diario de Ansay no menciona el quebracho blanco (Aspidosperma quebracho-blanco). Actualmente esta especie es abundante al este del río Desaguadero, y es la dominante o codominante en los bosques del área (Roig 1981). Tampoco se han registrado alusiones directas sobre esta especie entre los viajeros y naturalistas consultados. Solamente Samuel Greene Arnold (1951) escribe que en la zona de Balde $(25 \mathrm{~km}$ al oeste de la ciudad de San Luis) hay “...una variedad de acebo con corteza de corcho con hojas duras, gruesas y afiladas en esta forma..." (p. 203), descripción que se acerca a las características del quebracho. Esta especie no era desconocida para los españoles, que la bautizaron "quebrahacha" (ver T. Haenke 1794, f. 46 v). Ante esta ausencia de referencias expresas, se pueden proponer algunas interpretaciones: (a) el quebracho tendría un área de distribución similar a la actual, pero no habría sido percibida como recurso, una explicación que consideramos improbable; (b) las poblaciones de quebracho en esta zona no eran tan conspicuas y/o su área de distribución era lo suficientemente restringida como para pasar desapercibida. De ser correcta esta interpretación implicaría una posterior expansión del área de distribución, como resultado del aumento de precipitaciones y/o de su historia de uso.

Esta última interpretación es consistente con las evidencias sobre cambios climáticos y las distintas predicciones para la zona. Estas evidencias indican un incremento en las medias anuales de temperatura y humedad y el consiguiente co- rrimiento hacia el oeste de las isoyetas e isotermas (Hoffmann 1989). Como consecuencia se podría esperar un corrimiento hacia el oeste de los límites de distribución de algunas especies.

\section{LITERATURA CITADA}

ABRAHAM EM (2000) Geomorfología de la Provincia de Mendoza. En: Abraham EM \& F Rodríguez Martínez (eds) Argentina: recurso y problemas ambientales de la zona árida. Provincias de Mendoza, San Juan y La Rioja: 29-48. Junta de Gobierno de Andalucía, universidades y centros de investigación de la Región Andina Argentina, Mendoza, Argentina.

ABRAHAM EM \& MR PRIETO (1999) Vitivinicultura y desertificación en Mendoza. En: García-Martínez B (ed) Estudios de historia y ambiente en América: Argentina, Bolivia, México, Paraguay: 109-135. IPGH-Colegio de México, México.

BARON W (1982) The reconstruction of eighteenth century temperature records through the use of content analysis. Climate Change 4: 385-398.

BRADLEY RS (1985) Quaternary paleoclimatology: methods of paleoclimatic reconstruction. Allen and Unwin, Boston, Massachusetts, USA. 472 pp.

BRAUN RH, E DUFFAR, RJ CANDIA, MH PACHECO, HA ESTRELLA \& A BERRA (1984) Carta ecológica del sector extra-montano de la provincia de Mendoza (escala 1:200.000). Publicación Especial del IADIZA. Instituto Argentino de Investigaciones de Zonas Áridas, Mendoza, Argentina. 33 pp.

BUFFINGTON LC \& CH HERBEL (1965) Vegetational changes on a semidesert grassland range from 1858 to 1963. Ecological Monographs 35: 139-164.

CABIDO M, C GONZÁLEZ \& S DÍAZ (1993) Vegetation changes along a precipitation gradient in Central Argentina. Vegetatio 109: 5-14.

CALDCLEUGH A (1943) Viajes por América del Sur. Río de la Plata. 1821. Ediciones Argentinas "Solar", Buenos Aires, Argentina. 255 pp.

FONT QUER P \& E SIERRA RAFOLS (1979) Diccionario de botánica. Labor, Barcelona, España. 1.244 pp.

GREENE-ARNOLD S (1951) Viaje por América del Sur 1947-1848. Emecé Editorial, Buenos Aires, Argentina. $260 \mathrm{pp}$.

GUEVARA JC, JB CAVAGNARO, OR ESTÉVEZ, HN LE HOUÉROU \& CR STASI (1997) Productivity, management and development problems in the arid rangelands of the central Mendoza plains (Argentina). Journal of Arid Environments 35: 575-600.

HAENKE T (1794) Diario del viaje entre Chile y Buenos Aires. Archivo del Museo Naval, Sección Manuscritos, Madrid, España.

HOFFMANN JA (1989) Las variaciones climáticas ocurridas en la Argentina desde fines del siglo pasado hasta el presente. Centro para la Promoción de la Conservación del Suelo y del Agua (eds) El deterioro del ambiente en la Argentina: 275-290. PROSA, Buenos Aires, Argentina.

KENT M \& P COKER (1992) Vegetation description and analysis: a practical approach. CRC Press-Belhaven Press, London, United Kingdom. 363 pp.

LEMOS A (1888) Memoria descriptiva de la Provincia de Mendoza. Editorial Los Andes, Mendoza, Argentina. 287 pp.

MACRAE A (1856) Report of journeys across the Andes and Pampas of the Argentine Provinces. Lippincott \& Co, Philadelphia, Pennsylvania, USA. 
MANNION AM (1997) Global environmental change: a natural and cultural environmental history. Longman, New York, New York, USA. 387 pp.

MATTEUCCI SD \& A COLMA (1982) Metodología para el estudio de la vegetación. Secretaría General de la Organización de lo Estados Americanos, Washington, District of Columbia, USA. 168 pp.

MUHN J (1946) La Argentina vista por viajeros del siglo XVIII. Huarpes, Buenos Aires, Argentina. 159 pp.

PRIETO MR \& CF WUILLOUD (1986) Consecuencias ambientales derivadas de la instalación de los españoles en Mendoza en 1562. Cuadernos de Historia Regional. Universidad de Luján (Argentina) 2: 14-30.

PRIETO MR \& EM ABRAHAM (2000) Caminos y comercio como factores de cambio ambiental en las planicies áridas de Mendoza (Argentina) entre los siglos XVII y XIX. Revista Theomai, Estudios sobre Sociedad, Naturaleza y Desarrollo: http:// www.unq.edu.ar/revista-theomai/numero2/ index.htm.

REAL ACADEMIA ESPAÑOLA (1791) Diccionario de la lengua castellana compuesto por la Real Academia Española reducido a un tomo para su más fácil uso. Viuda de Don Joaquín Ibarra, Impresora de la Real Academia, Madrid, España.
ROIG FA (1971) Flora y vegetación de la Reserva Forestal de Ñacuñán. La vegetación. Deserta (Argentina) 1: 201-239.

ROIG FA (1981) Conservación de la vegetación natural en Cuyo. Serie Científica (Mendoza, Argentina) 25: 36-39.

ROIG FA \& BE ROSSI (2001) Flora y vegetación. En: Claver S \& SA Roig-Juñent (eds) El desierto del Monte: la Reserva de Biósfera de Ñacuñán: 41-70. IADIZA-MAB- UNESCO, Mendoza, Argentina.

SCHMIDTMEYER P (1947) Viaje a Chile a través de los Andes. Editorial Claridad, Buenos Aires, Argentina. $350 \mathrm{pp}$.

SKOLE D \& C TUCKER (1993) Tropical deforestation and habitat fragmentation in the Amazon: satellite data from 1978 to 1988. Science 260: 1905-1910.

WOLTERS V, WL SILVER, DE BIGNELL, DC COLEMAN, $P$ LAVELLE, WH VAN DER PUTTEN, P DE RUITER, J RUSEK, DH WALL, DA WARDLE, L BRUSSAARD, JM DANGERFIELD, VK BROWN, KE GILLER, DU HOOPER, O SALA, J TIEDJE \& JA VAN VEEN (2000) Effects of Global Changes on Above- and belowground biodiversity in terrestrial ecosystems: implications for ecosystem functioning. BioScience 50: 1089-1099. 